Math. Ann. 307, 315-324 (1997)

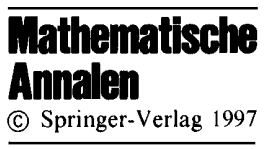

\title{
Invariants from an analytic function on hyperbolic Dehn surgery space
}

\author{
Mingqing Ouyang \\ Department of Mathematics, University of Michigan, Ann Arbor, MI 48109, USA \\ (e-mail mouyang@math.lsa.umich.edu)
}

Received: 11 November 1995/Revised version: 13 February 1996

\section{Introduction}

Let $M$ be a complete finite-volume hyperbolic 3-manifold with cusps. Then $M$ has an ideal triangulation

$$
M=\Delta\left(z_{1}\right) \cup \cdots \cup \Delta\left(z_{n}\right)
$$

where each $\Delta\left(z_{\lambda}\right)$ is an ideal tetrahedron in $\mathbf{H}^{3}$ described by a complex number $z_{\lambda}$ (the tetrahedral parameter) ([EP] and $[\mathrm{T}]$ ).

There are several invariants one can get from an ideal triangulation of $M$. The most notable one is the volume of $M$ which is the sum of the volumes of $\Delta\left(z_{v}\right)$. Another invariant is $k_{\Delta}(M)$, the field over the rationals generated by the tetrahedral parameters of an ideal triangulation of $M$. As shown in [NR1], if $M=\mathbf{H}^{3} / \Gamma$ where $\Gamma$ is a Kleinian group of finite covolume, then $k_{\Delta}(M)$ coincides with the invariant trace field of $\Gamma$ generated by $\left\{\operatorname{tr}\left(\gamma^{2}\right): \gamma \in \Gamma\right\}$. This justifies the well-definedness of $k_{\Delta}(M)$.

In general, there are many different ways one can glue together given $n$ ideal tetrahedra to form distinct 3-manifolds. It is clear that neither the volume nor $k_{\Delta}(M)$ depends on gluing patterns.

In this paper we show that a sequence of subfields of $k_{\Delta}(M)$ are welldefined invariants of $M$ which reflect the gluing pattern of an ideal triangulation of $M$. The invariants are extracted from an analytic function defined on hyperbolic Dehn surgery space. Such an analytic function, studied first in [NZ], is closely related with the way the structure of the cusps varies as the hyperbolic structure on $M$ is deformed. Moreover, the corresponding variation of the analytic function reveals a complex-analytic relation between the volume and the Chern-Simons invariant of a hyperbolic structure on $M$ ([NZ] and [Y]).

The definition of the analytic function depends on the choice of a meridianlongitude pair at each cusp. For each $n \in \mathbf{Z}^{+}$, denote by $\mathscr{F}_{n}$ the field (over the rational $\mathbf{Q}$ ) generated by the coefficients of the terms with total degree no 
greater than $2 n$ in the Taylor series of the analytic function. It turns out that each $\mathscr{F}_{n}$ is a well-defined invariant of $M$, indeed an invariant of the commensurability class of $M$. We call $\mathscr{F}_{n}$ it the $n$-th cusp field of $M$. Furthermore we show that each $\mathscr{F}_{n}$ lies in $k_{\Delta}(M)$, giving the sequence of the subfields of $k_{\Delta}(M)$.

Suppose that $M$ is the complement of a hyperbolic knot or link $L$ in a homology sphere. Then the analytic function determined by the topological meridian and longitude of each component of $L$ is an invariant of $L$. Thus the coefficients in the Taylor expansion of the analytic function give rise to a sequence of link invariants. For a general cusped hyperbolic 3-manifold $M$, one could choose meridian and longitude to correspond to the parabolic isometries which are two shortest linearly independent translations in each cusp torus. The analytic function with respect to such a choice defines an invariant of $M$.

The analytic function and the cusp fields can be computed from an ideal triangulation of $M$ and the associated gluing equations readily available in Jeff Weeks' "Snappea" program. The proof of a proposition in Sect. 3 displays how the calculations proceed.

Given a knot in $S^{3}$. A new knot can be obtained by taking out a tangle, flipping it over and gluing it back in. Such an operation is called mutation. The resulting knot tends to differ from the original one. It is well known that a pair of mutant knot are hard to distinguish. Indeed nearly all known invariants fail to tell them apart.

In this note, we show that the corresponding coefficients in the Taylor expansions of the analytic functions associated with a pair of mutant hyperbolic knots coincide.

We organize this note as follows. Section 2 reviews some preliminary material from [T] and [NZ]. We define the cusp fields of $M$ in Sect. 3 and go on to discuss some properties of these fields. In Sect. 4 we show that the analytic function, as a link invariant, can be used to distinguish many links with homeomorphic complements. We also prove that mutation preserves the corresponding coefficients in the Taylor expansions of the analytic functions of a pair of mutant hyperbolic knots.

\section{Deformation space of hyperbolic structure and the analytic function}

Suppose that $M$ is a complete hyperbolic 3-manifold of finite volume with $h$ cusps. Then $M$ has at least one ideal triangulation ([EP])

$$
M=\Delta_{1} \cup \cdots \cup \Delta_{n}
$$

where each $\Delta_{\lambda}$ is an ideal tetrahedron in $\mathbf{H}^{3}$.

As described in [T], after fixing an edge, each ideal tetrahedron $\Delta_{\lambda}$ can be described up to isometry by a complex number $z_{\lambda}^{0}$ with positive imaginary part such that the Euclidean triangle cut off at any vertex of $\Delta_{\lambda}$ by a horosphere section is similar to the triangle with vertices at $0,1, z_{\lambda}^{0}$ in the complex plane. 
Associated to an ideal triangulation is a system of gluing consistency relations of the form

$$
\prod_{\lambda=1}^{n}\left(z_{\lambda}^{0}\right)^{r^{\prime}}{ }^{\prime}\left(1-z_{\lambda}^{0}\right)^{r_{v \lambda}^{\prime \prime}}= \pm 1, \quad v=1, \ldots, n
$$

where $r_{v \lambda}^{\prime}$ and $r_{v \lambda}^{\prime \prime}$ are integers.

By deforming each $\Delta\left(z_{\lambda}^{0}\right)$ to $\Delta\left(z_{\lambda}\right)$ with $\operatorname{Im}\left(z_{\lambda}\right)>0$ and keeping the same gluing pattern one obtains an incomplete hyperbolic structure on $M$. Thurston's deformation space $\mathscr{D}(M)$ of the hyperbolic structure on $M$ is the variety of $\left(z_{1}, \ldots, z_{n}\right) \in \mathbf{C}^{n}$ satisfying the following gluing consistency relations

$$
\prod_{\lambda=1}^{n} z_{\lambda}^{r_{\nu \lambda}^{\prime}}\left(1-z_{\lambda}\right)^{r_{v \lambda}^{\prime \prime}}= \pm 1 \quad v=1, \ldots, n
$$

$\mathscr{D}(M)$ is also termed as the hyperbolic surgery space of $M$.

For each $j=1, \ldots, h$, fix a meridian-longitude basis $\left(\mathbf{m}_{j}, \mathbf{l}_{j}\right)$ for the homology of the torus $T_{j}$ corresponding to a horospherical cross-section of cusp $j$. Denote by $u_{j}$ (resp. $v_{j}$ ) twice the logarithm of an eigenvalue of the holonomy of $\mathbf{m}_{j}\left(\right.$ resp. $\left.\mathbf{l}_{j}\right)$. By $[\mathrm{T}]$ and [NZ], $\mathscr{D}(M)$ has complex dimension $h$ and can be holomorphically parameterized by $\mathbf{u}=\left(u_{1}, \ldots, u_{h}\right) \in \mathbf{C}^{h}$ in a neighborhood of the origin in $\mathbf{C}^{h}$. In what follows, we will denote by $M_{\mathbf{u}}$ the manifold $M$ with the hyperbolic structure parametrized by some $\mathbf{u} \in \mathscr{D}(M)$.

The following theorem was proved in [NZ]

Theorem 2.1. In a neighborhood of the origin in $\mathbf{C}^{h}$

(1) $v_{j}=u_{j} \cdot \tau_{j}\left(u_{1}, \ldots, u_{h}\right)$ where each $\tau_{j}$ is an even function of its arguments;

(2) There is an analytic function $\Phi\left(u_{1}, \ldots, u_{h}\right)$ such that $\partial \Phi / \partial u_{j}=2 v_{j}$ and $\Phi(0, \ldots, 0)=0$.

(3) $\Phi\left(u_{1}, \ldots, u_{h}\right)$ is even in each argument and can be written as

$$
\Phi\left(u_{1}, \ldots, u_{h}\right)=\int_{0}^{\mathbf{u}} \sum_{j=1}^{h}\left(v_{j} d u_{j}-u_{j} d v_{j}\right)+\sum_{j=1}^{h} u_{j} v_{j} .
$$

Let $\tau_{j}$ be the modulus of the Euclidean structure of $T_{j}$ with respect to $\left(\mathbf{m}_{j}, \mathbf{l}_{j}\right)$. Then $\Phi$ has the Taylor expansion

$$
\Phi\left(u_{1}, \ldots, u_{h}\right)=\tau_{1} u_{1}^{2}+\cdots+\tau_{1} u_{h}^{2}+\text { (higher order) } .
$$

As shown in [NZ], if $f(\mathbf{u})$ is the variation of $\Phi(\mathbf{u})$ defined by

$$
f(\mathbf{u})=\frac{1}{4} \int_{0}^{\mathbf{u}} \sum_{j=1}^{h}\left(v_{j} d u_{j}-u_{j} d v_{j}\right)
$$

then

$$
\operatorname{Vol}\left(M_{\mathbf{u}}\right)=\operatorname{Vol}(M)+\operatorname{Im} f(\mathbf{u})-\sum_{j=1}^{h} \frac{1}{4} \operatorname{Im}\left(\bar{u}_{j} v_{j}\right)
$$

where 'bar' denotes the complex conjugation. As conjectured in [NZ] and proven in [Y], when the metric completion $\bar{M}_{\mathbf{u}}$ of $M_{\boldsymbol{u}}$ is the result of 
a hyperbolic Dehn surgery on $M$,

$$
2 C S\left(\bar{M}_{\mathbf{u}}\right)=2 C S(M)-\frac{1}{2 \pi} \sum_{k} \operatorname{Torsion}\left(\lambda_{k}\right)-\frac{1}{\pi^{2}} \operatorname{Re} f(\mathbf{u}) \quad(\bmod \mathbf{Z})
$$

where $\lambda_{k}$ is the core geodesic of the filled-in solid torus at a surgered cusp.

\section{The cusp fields of $M$}

Let $M$ be a complete finite-volume hyperbolic 3-manifold with $h$ cusps. Let $\Phi(\mathbf{u})$ be the analytic function with respect to a meridian-longitude basis with the Taylor expansion

$$
\Phi(\mathbf{u})=\sum_{n_{1}, \ldots, n_{h}=1}^{\infty} C_{n_{1} \cdots n_{h}} u_{1}^{n_{1}} \cdots u_{h}^{n_{h}} .
$$

For each $n \in \mathbf{Z}^{+}$define $\mathscr{F}_{n}:=\mathbf{Q}\left(C_{n_{1} \cdots n_{h}} ; n_{1}+\cdots+n_{h} \leqq 2 n\right)$. Thus one has a tower of fields $\mathscr{F}_{1} \subseteq \mathscr{F}_{2} \subseteq \ldots$ with $\mathscr{F}_{1}=\mathbf{Q}\left(\tau_{1}, \ldots, \tau_{h}\right), \cdots$

Proposition 3.1. Each $\mathscr{F}_{n}$ is an invariant of $M$.

Proof. Let $\left(\mathbf{m}_{j}, \mathbf{l}_{j} ; j=1, \ldots, h\right)$ and $\left(\dot{\mathbf{m}}_{j}, \dot{\mathbf{I}}_{j} ; j=1, \ldots, h\right)$ be two meridianlongitude bases. Then one has $\dot{\mathbf{m}}_{j}=\alpha_{j} \mathbf{m}_{j}+\beta_{j} \mathbf{l}_{j}$ and $\dot{\mathbf{I}}_{j}=\gamma_{j} \mathbf{m}_{j}+\delta_{j} \mathbf{l}_{j}$ where $\left(\begin{array}{ll}\alpha_{j} & \beta_{j} \\ \gamma_{j} & \delta_{j}\end{array}\right) \in S L_{2}(\mathbf{Z})$. For each $1 \leqq j \leqq h$ let $v_{j}=\sum c_{j, n_{1} \cdots n_{h}} u_{1}^{n_{1}} \cdots u_{h}^{n_{h}}$ and $\dot{v}_{j}=\sum \dot{c}_{j, n_{1} \cdots n_{h}} \dot{u}_{1}^{n_{1}} \cdots \dot{u}_{h}^{n_{h}}$. Then $\dot{u}_{j}=\alpha_{j} u_{j}+\beta_{j} v_{j}$ and $\dot{v}_{j}=\gamma_{j} u_{j}+\delta_{j} v_{j}$. By substituting these into the series for $\dot{v}_{j}$ and comparing the coefficients of $u^{n_{1}} \cdots u^{n_{h}}$ in both sides of the equation one sees inductively that $\dot{c}_{j, n_{1} \cdots n_{h}} \in \mathbf{Q}\left(c_{s, t_{1} \cdots t_{h}} ; s \leqq\right.$ $\left.h, t_{1}+\cdots+t_{h} \leqq n_{1}+\cdots+n_{h}\right)$. Proposition 3.1 follows from (2.2). Q.E.D.

Thus it is clear that each $\mathscr{F}_{n}$ is indeed an invariant of the commensurability class of $M$. We call it the $n$-th cusp field of $M$.

Suppose that $M=\Delta\left(z_{1}^{0}\right) \cup \cdots \cup \Delta\left(z_{n}^{0}\right)$ is an ideal triangulation of $M$. Define $k_{\Delta}(M):=\mathbf{Q}\left(z_{1}^{0}, \ldots, z_{n}^{0}\right)$. A priori $k_{\Delta}(M)$ might depend on the choice of ideal triangulation. But as shown in [NR1], if $M=\mathbf{H}^{3} / \Gamma$ where $\Gamma$ is a Kleinian group of finite covolume, then $k_{\Delta}(M)$ coincides with the invariant trace field $\left.k(\Gamma):=\mathbf{Q}\left((\operatorname{tr} \gamma)^{2}: \gamma \in \Gamma\right\}\right)$. It follows that $k_{\Delta}(M)$ is an invariant of $M$, indeed an invariant of the commensurability class of $\Gamma$.

Proposition 3.2. Each $\mathscr{F}_{n}$ is a subfield of $k_{\Delta}(M)$, hence a subfield of $k(\Gamma)$.

Proof. By virtue of (2.2) it is enough to show that each $c_{j, n_{1} \cdots n_{h}}$ lies in $\mathbf{Q}\left(z_{1}^{0}, \ldots, z_{n}^{0}\right)$.

In the Taylor expansion for each $v_{j}$ one has

$$
c_{k, n_{1} \cdots n_{h}}=\left.\frac{1}{n_{1} ! \cdots n_{h} !} \frac{\partial^{n_{1}+\cdots+n_{h}} v_{k}}{\partial u_{1}^{n_{1}} \cdots \partial u_{h}^{n_{h}}}\right|_{0} .
$$

Therefore it suffices to show that the latter is contained in $\mathbf{Q}\left(z_{1}^{0}, \ldots, z_{n}^{0}\right)$. 
As in [NZ] one has

$$
u_{k}=\sum_{\lambda=1}^{n}\left(m_{k \lambda}^{\prime} \log \frac{z_{\lambda}}{z_{\lambda}^{0}}+m_{k \lambda}^{\prime \prime} \log \frac{1-z_{\lambda}}{1-z_{\lambda}^{0}}\right) \quad k=1, \ldots, h
$$

and

$$
v_{k}=\sum_{\lambda=1}^{n}\left(l_{k \lambda}^{\prime} \log \frac{z_{\lambda}}{z_{\lambda}^{0}}+l_{k \lambda}^{\prime \prime} \log \frac{1-z_{\lambda}}{1-z_{\lambda}^{0}}\right) \quad k=1, \ldots, h
$$

where $m_{k \lambda}^{\prime}, m_{k \lambda}^{\prime \prime}, l_{k \lambda}^{\prime}$ and $l_{k \lambda}^{\prime \prime} \in \mathbf{Z}$.

The gluing consistency relations $(2.1)$ can be rewritten as

$$
\sum_{\lambda=1}^{n}\left(r_{v \lambda}^{\prime} \log z_{\lambda}+r_{v \lambda}^{\prime \prime} \log \left(1-z_{\lambda}\right)\right)=\pi i d_{v} \quad v=1, \ldots, n
$$

where $r_{v \lambda}^{\prime}, r_{v \lambda}^{\prime \prime}$ and $d_{v} \in \mathbf{Z}$.

By taking partial derivative with respect to $u_{j}$ in (3.1) and (3.3) one gets

$$
\begin{aligned}
\sum_{\lambda=1}^{n}\left(\frac{m_{k \lambda}^{\prime}}{z_{\lambda}}-\frac{m_{k \lambda}^{\prime \prime}}{1-z_{\lambda}}\right) \frac{\partial z_{\lambda}}{\partial u_{j}} & =1 \quad \text { if } j=k \\
& =0 \text { if } j \neq k \quad k=1, \ldots, h
\end{aligned}
$$

and

$$
\sum_{\lambda=1}^{n}\left(\frac{r_{v \lambda}^{\prime}}{z_{\lambda}}-\frac{r_{v \lambda}^{\prime \prime}}{1-z_{\lambda}}\right) \frac{\partial z_{\lambda}}{\partial u_{j}}=0 . \quad v=1, \ldots, n
$$

Let

$$
\begin{aligned}
\mathscr{D}^{\prime}(M) & =\left\{\mathbf{z}=\left(z_{1}, \ldots, z_{n}\right) \in \mathscr{D}(M)|\operatorname{dim}(\mathscr{D}(M))|_{\mathbf{z}}\right. \\
& =n-h, \mathscr{D}(M) \text { is nonsingular at } \mathbf{z}\} .
\end{aligned}
$$

As shown in $[\mathrm{NZ}],\left(z_{1}^{0}, \ldots, z_{n}^{0}\right) \in \mathscr{D}^{\prime}(M)$. Notice that

$$
\mathscr{I}=\left(\begin{array}{ccc}
r_{11}^{\prime}-\frac{r_{11}^{\prime \prime}}{z_{1}} & \ldots & \frac{r_{1 n}^{\prime}}{z_{n}}-\frac{r_{1 n}^{\prime \prime}}{1-z_{n}} \\
\vdots & \vdots & \vdots \\
\frac{r_{n 1}^{\prime}}{z_{1}}-\frac{r_{n 1}^{\prime \prime}}{1-z_{1}} & \ldots & \frac{r_{n n}^{\prime}}{z_{n}}-\frac{r_{n n}^{\prime \prime}}{1-z_{n}}
\end{array}\right)
$$

is the Jacobian of the system (3.3). Thus for each $\mathbf{z}=\left(z_{1}, \ldots, z_{n}\right) \in \mathscr{D}^{\prime}(M)$, $\operatorname{Rank}(\mathscr{I})=n-h$.

Without loss of generality, we assume that the submatrix

$$
\mathscr{I}^{\prime}=\left(\begin{array}{ccc}
r_{11}^{\prime}-\frac{r_{11}^{\prime \prime}}{z_{1}} & \ldots & \frac{r_{1 n}^{\prime}}{z_{n}}-\frac{r_{1 n}^{\prime \prime}}{1-z_{n}} \\
\vdots & \vdots & \vdots \\
\frac{r_{(n-h) 1}^{\prime}}{z_{1}}-\frac{r_{(n-h) 1}^{\prime \prime}}{1-z_{1}} & \ldots & \frac{r_{(n-h) n}^{\prime}}{z_{n}}-\frac{r_{(n-h) n}^{\prime \prime}}{1-z_{n}}
\end{array}\right)
$$

has rank $n-h$. 
Denote

$$
\mathscr{J}=\left(\begin{array}{cccc}
\frac{m_{11}^{\prime}}{z_{1}}-\frac{m_{11}^{\prime \prime}}{1-z_{1}} & \ldots & \frac{m_{1 n}^{\prime}}{z_{n}}-\frac{m_{1 n}^{\prime \prime}}{1-z_{n}} \\
\vdots & \vdots & \vdots \\
\frac{m_{h 1}^{\prime}}{z_{1}}-\frac{m_{h 1}^{\prime \prime}}{1-z_{1}} & \ldots & \frac{m_{h n}^{\prime}}{z_{n}}-\frac{m_{h n}^{\prime \prime}}{1-z_{n}}
\end{array}\right) .
$$

Then it follows from (3.4) and (3.5) that $\operatorname{Rank}\left(\begin{array}{c}\mathscr{I}^{\prime} \\ \mathscr{J}\end{array}\right)=n$.

Thus the system of the linear equations consisting of (3.4) and

$$
\sum_{\lambda=1}^{n}\left(\frac{r_{v \lambda}^{\prime}}{z_{\lambda}}-\frac{r_{v \lambda}^{\prime \prime}}{1-z_{\lambda}}\right) \frac{\partial z_{\lambda}}{\partial u_{j}}=0 . \quad v=1, \ldots, n-h
$$

is independent and has a unique solution

$$
\frac{\partial z_{1}}{\partial u_{j}}, \ldots, \frac{\partial z_{n}}{\partial u_{j}} \in \mathbf{Q}\left(z_{1}, \ldots, z_{n}\right) .
$$

Since

$$
\frac{\partial v_{k}}{\partial u_{j}}=\frac{\partial v_{k}}{\partial z_{1}} \frac{\partial z_{1}}{\partial u_{j}}+\cdots+\frac{\partial v_{k} \partial z_{n}}{\partial z_{n} \partial u_{j}}
$$

it follows from (3.2) and (3.6) that

$$
\frac{\partial v_{k}}{\partial u_{j}} \in \mathbf{Q}\left(z_{1}, \ldots, z_{n}\right) .
$$

By induction one concludes that

$$
\frac{\partial^{n_{1}+\cdots+n_{h}} v_{k}}{\partial u_{1}^{n_{1}} \cdots \partial u_{h}^{n_{h}}} \in \mathbf{Q}\left(z_{1}, \ldots, z_{n}\right)
$$

giving the result.

Q.E.D.

Remark. It is natural to ask whether each $\mathscr{F}_{n}$ is a proper subfield of $k_{\Delta}(M)=$ $k(\Gamma)$. As pointed out in [NR1], for hyperbolic knot complements, the only known examples where $\mathscr{F}_{1}=\mathbf{Q}\left(\tau_{1}, \ldots, \tau_{h}\right)$ is not equal to $k(\Gamma)$ are the two dodecahedral knots of Aitchison and Rubinstein [AR]. In contrast to the case of knot complements, they are many 1-cusp orbifolds with $\mathscr{F}_{1}$ smaller than the invariant trace field. Nevertheless the following conjecture seems to be reasonable.

Conjecture 3.3. The direct limit of $\mathscr{F}_{n}$ is equal to $k_{\Delta}(M)$.

\section{The analytic function as a link invariant}

Suppose that $M$ is the complement of a hyperbolic knot or link $L$ in an integer homology sphere. Then one can choose meridian and longitude curves at each cusp of $M$ to be the topological meridian and longitude of each component of $L$. Thus the analytic function $\Phi$ determined by this canonical choice defines a link 


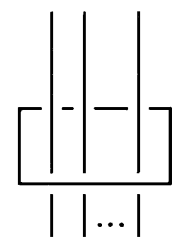

(a)

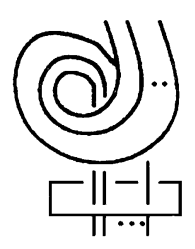

(b)

Fig. 1.

invariant $\Phi_{L}$. Such an invariant can be used to distinguish many links with homeomorphic complements.

Let $L$ be a hyperbolic link in $S^{3}$ with $n$ components whose projection contains a portion as shown in Fig. 1a.

Denote by $D$ the punctured disk bounded the trivial component $L_{1}$. By cutting $S^{3}-L$ open along $D$, twisting it a full twist and reidentifying, one obtains a link $L^{\prime}$ containing a portion as in Fig. $1 \mathrm{~b}$ whose complement is homeomorphic to that of $L$.

Theorem 4.1. $L^{\prime}$ is never equivalent to $L$.

Proof. We will use the coefficients of the second degree terms in the Taylor expansions of $\Phi_{L}$ and $\Phi_{L^{\prime}}$ to tell them apart.

Let $L=L_{1} \cup \cdots \cup L_{n}$ and $L^{\prime}=L_{1}^{\prime} \cup \cdots \cup L_{n}^{\prime}$ where $L_{1}$ and $L_{1}^{\prime}$ are the trivial components. Then a homeomorphism between $S^{3}-L$ and $S^{3}-L^{\prime}$ is given by a full twist $t$ on the solid torus $D^{2} \times S^{1} \cong S^{3}-L_{1} \cong S^{3}-L_{1}^{\prime}$ which sends $L_{2} \cup$ $\cdots \cup L_{n}$ to $L_{2}^{\prime} \cup \cdots \cup L_{n}^{\prime}$. By Mostow-Prasad rigidity theorem, $t$ is homotopic to a hyperbolic isometry $t^{\prime}$ between the complete, finite-volume hyperbolic manifolds $S^{3}-L$ and $S^{3}-L^{\prime}$. Let $\lambda_{i}$ be the linking number between $L_{1}$ and $L_{i}$. Denote by $\left(m_{i}, l_{i}\right)$ and $\left(m_{i}^{\prime}, l_{i}^{\prime}\right)$ the topological meridian-longitude pairs of the $i$-th component of $L$ and $L^{\prime}$. It is not hard to see (refer to [Ro] for instance) that $t$ (hence $t^{\prime}$ ) sends $m_{1}$ and $l_{1}$ to $m_{1}^{\prime}+l_{1}^{\prime}$ and $l_{1}^{\prime}, m_{i}$ and $l_{i}$ to $m_{i}^{\prime}$ and $l_{i}^{\prime}+\sigma_{i}^{2} m_{i}^{\prime}$ for $i=2, \ldots, n$.

Identifying $S^{3}-L$ with $S^{3}-L^{\prime}$ via the hyperbolic isometry $t^{\prime}$, one can think of $\left(m_{i}, l_{i}\right)$ and $\left(m_{i}^{\prime}, l_{i}^{\prime}\right)$ as two different choices of meridian and longitude bases for the same manifold. As before, let $u_{i}$ and $v_{i}$ (resp. $u_{i}^{\prime}$ and $v_{i}^{\prime}$ ) be twice the logarithm of an eigenvalue of the holonomy of $m_{i}$ and $l_{i}$ (resp. $m_{i}^{\prime}$ and $l_{i}^{\prime}$ ). Then one has

$$
u_{1}=u_{1}^{\prime}+v_{1}^{\prime}, \quad v_{1}=v_{1}^{\prime}
$$

and

$$
u_{i}=u_{i}^{\prime}, \quad v_{i}=v_{i}^{\prime}+\sigma_{i}^{2} u_{i}^{\prime} \text { for } i=2, \ldots, n .
$$

In what follows, we denote by $\tau_{i}$ (resp. $\tau_{i}^{\prime}$ ) the modulus of the Euclidean structure of the torus cusp corresponding to the $i$-th component of $L$ (resp. $L^{\prime}$ ). 
We have

$$
\tau_{1}=\left.\frac{v_{1}}{u_{1}}\right|_{(0, \ldots, 0)}=\frac{\left.\begin{array}{c}
v_{1}^{\prime} \\
u_{1}^{\prime}
\end{array}\right|_{(0, \ldots, 0)}}{1+\left.\begin{array}{c}
v_{1}^{\prime} \\
u_{1}^{\prime}
\end{array}\right|_{(0, \ldots, 0)}}=\frac{\tau_{1}^{\prime}}{1+\tau_{1}^{\prime}}
$$

and

$$
\tau_{i}=\left.\frac{v_{i}}{u_{i}}\right|_{(0, \ldots, 0)}=\left.\begin{gathered}
v_{i}^{\prime} \\
u_{i}^{\prime}
\end{gathered}\right|_{(0, \ldots, 0)}+\sigma_{i}^{2}=\tau_{i}^{\prime}+\sigma_{i}^{2} \quad \text { for } i=2, \ldots, n .
$$

To prove that $L$ is not equivalent to $L^{\prime}$, it suffices to show that the two tubes $\left(\tau_{1}, \tau_{2}, \ldots, \tau_{n}\right)$ and $\left(\tau_{1}^{\prime}, \tau_{2}^{\prime}, \ldots, \tau_{n}^{\prime}\right)$ are not the same up to permutations.

Suppose that they were, then we would have

$$
\frac{\tau_{1}^{\prime}}{1+\tau_{1}^{\prime}}+\left(\tau_{2}^{\prime}+\sigma_{2}^{2}\right)+\cdots+\left(\tau_{n}^{\prime}+\sigma_{n}^{2}\right)=\tau_{1}^{\prime}+\tau_{2}^{\prime}+\cdots+\tau_{n}^{\prime}
$$

which would imply

$$
\tau_{1}^{\prime}=\frac{\tau_{1}^{\prime}}{1+\tau_{1}^{\prime}}+\sigma_{2}^{2}+\cdots+\sigma_{n}^{2}
$$

and hence

$$
\tau_{1}^{\prime}=\frac{\sum_{i=2}^{n} \sigma_{i}^{2} \pm \sqrt{\left(\sum_{i=2}^{n} \sigma_{i}^{2}\right)^{2}+4\left(\sum_{i=2}^{n} \sigma_{i}^{2}\right)}}{2} .
$$

Thus $\tau_{1}$ would be a real number.

Since $\tau_{1}^{\prime}$ is the modulus of the Euclidean structure of the cusp torus corresponding to the trivial component of $L^{\prime}$, it can not be a real number. This contradiction completes our proof of Theorem 4.1.

Q.E.D.

Suppose that $K$ is a hyperbolic knot in $S^{3}$ and $K^{\mu}$ a mutant of $K$. Then $K^{\mu}$ is hyperbolic $([\mathrm{Ru}])$. Let $m$ and $l$ be the topological meridian and longitude of $K$. It is not hard to see that the linking number between $\mu(l)$ and $K^{\mu}$ is 0 . Thus $\mu(m)$ and $\mu(l)$ form the topological meridian and longitude of $K^{\mu}$. Denote $M=S^{3}-K$ and $M^{\mu}=S^{3}-K^{\mu}$.

Let $\Phi$ and $\tilde{\Phi}$ be the analytic functions for $K$ and $K^{\mu}$ with Taylor expansions

$$
\Phi(u)=\sum_{n=1}^{\infty} C_{n} u^{n} \quad \text { and } \quad \tilde{\Phi}(\tilde{u})=\sum_{n=1}^{\infty} \tilde{C}_{n} \tilde{u}^{n} .
$$

Theorem 4.2. $C_{n}=\tilde{C}_{n}$ for all $n \in \boldsymbol{Z}^{+}$.

Proof. Let

$$
v=\sum_{n=1}^{\infty} c_{n} u^{n} \quad \text { and } \quad \tilde{v}=\sum_{n=1}^{\infty} \tilde{c}_{n} \tilde{u}^{n} .
$$

Since $\partial \Phi(u) / \partial u=2 v$ and $\partial \Phi(\tilde{u}) / \partial \tilde{u}=2 \tilde{v}$, it suffices to show that $c_{n}=\tilde{c}_{n}$ for all $n \in \mathbf{Z}^{+}$. 
By combining (2.3) and (2.4) one gets

$$
\operatorname{Vol}\left(M_{u}\right)=\operatorname{Vol}(M)+\frac{1}{4} \operatorname{Im}\left(\int_{0}^{u}(v d u-u d v)-\bar{u} v\right),
$$

which shows that $\operatorname{Vol}\left(M_{u}\right)$ is a real-analytic function on $\mathscr{D}(M)$.

As shown in $[\mathrm{Ru}], \operatorname{Vol}\left(M^{\mu}\right)=\operatorname{Vol}(M)$. Furthermore if $u$ (resp. $\left.\tilde{u}\right)$ parametrizes a true hyperbolic $(p, q)$-surgery on $M$ (resp. $\left.M^{\mu}\right)$, i.e., $(p, q)$ is either a coprime integer pair or $\infty$, then $\operatorname{Vol}\left(M_{\tilde{u}}^{\mu}\right)=\operatorname{Vol}\left(M_{u}\right)$. Since the set of true hyperbolic Dehn surgeries is dense in $\mathscr{D}(M)$ and $\operatorname{Vol}\left(M_{\mathbf{u}}\right)$ is real-analytic, one concludes that $\operatorname{Vol}\left(M_{\tilde{u}}^{\mu}\right)=\operatorname{Vol}\left(M_{u}\right)$ throughout $\mathscr{D}(M)$ and $\mathscr{D}\left(M^{\mu}\right)$. Thus it follows from (4.1) that for every $u \in \mathscr{D}(M)$ and $\tilde{u} \in \mathscr{D}\left(M^{\mu}\right)$

$$
\operatorname{Im}\left(\int_{0}^{\tilde{u}}(\tilde{v} d \tilde{u}-\tilde{u} d \tilde{v})-\overline{\tilde{u}} \tilde{v}\right)=\operatorname{Im}\left(\int_{0}^{u}(v d u-u d v)-\bar{u} v\right)
$$

which implies

$$
\begin{aligned}
& \operatorname{Im}\left(\sum_{n=1}^{\infty} \frac{n-1}{n+1} \tilde{c}_{n} \tilde{u}^{n+1}\right)-\operatorname{Im}\left(\sum_{n=1}^{\infty} \tilde{c}_{n} \tilde{u}^{n} \overline{\tilde{u}}\right) \\
& \quad=\operatorname{Im}\left(\sum_{n=1}^{\infty} \frac{n-1}{n+1} c_{n} u^{n+1}\right)-\operatorname{Im}\left(\sum_{n=1}^{\infty} c_{n} u^{n} \bar{u}\right) .
\end{aligned}
$$

Set $q=0$. Then the equations $p u+q v=2 \pi i$ and $p \tilde{u}+q \tilde{v}=2 \pi i$ imply

$$
u=\frac{2 \pi i}{p} \quad \text { and } \quad \tilde{u}=\frac{2 \pi i}{p} .
$$

It follows that

$$
v=\sum_{n=1}^{\infty} c_{n}\left(\frac{2 \pi i}{p}\right)^{n} \quad \text { and } \quad \tilde{v}=\sum_{n=1}^{\infty} \tilde{c}_{n}\left(\frac{2 \pi i}{p}\right)^{n} .
$$

By substituting (4.3) into (4.2) and comparing the corresponding coefficients in both sides one gets $\operatorname{Im}\left(c_{n}\right)=\operatorname{Im}\left(\tilde{c}_{n}\right)$.

Next one has the inverse expansions

$$
\begin{aligned}
u=\sum_{n=1}^{\infty} a_{n} v^{n} \text { with } a_{1} & =\frac{1}{c_{1}}, a_{3}=-\frac{c_{3}}{c_{1}^{4}}, a_{5}=\frac{3 c_{3}^{2}}{c_{1}^{7}}-\frac{c_{5}}{c_{1}^{6}}, \\
a_{7} & =-\left(\frac{12 c_{3}^{3}}{c_{1}^{10}}-\frac{8 c_{3} c_{5}}{c_{1}}+\frac{c_{7}}{c_{1}^{8}}\right), \ldots
\end{aligned}
$$

and

$$
\begin{aligned}
\tilde{u}=\sum_{n=1}^{\infty} \tilde{a}_{n} \tilde{v}^{n} \text { with } \tilde{a}_{1} & =\frac{1}{\tilde{c}_{1}}, \tilde{a}_{3}=-\frac{\tilde{c}_{3}}{\tilde{c}_{1}^{4}}, \tilde{a}_{5}=\frac{3 \tilde{c}_{3}^{2}}{\tilde{c}_{1}^{7}}-\frac{\tilde{c}_{5}}{\tilde{c}_{1}^{6}}, \\
\tilde{a}_{7} & =-\left(\frac{12 \tilde{c}_{3}^{3}}{\tilde{c}_{1}^{10}}-\frac{8 \tilde{c}_{3} \tilde{c}_{5}}{\tilde{c}_{1}}+\frac{\tilde{c}_{7}}{\tilde{c}_{1}^{8}}\right), \ldots
\end{aligned}
$$


Set $p=0$. Using the inverse expansions the same argument as above yields $\operatorname{Im}\left(a_{n}\right)=\operatorname{Im}\left(\tilde{a}_{n}\right)$.

Using (4.4) and (4.5) with the fact that $\operatorname{Im}\left(c_{1}\right) \neq 0$ and $\operatorname{Im}\left(\tilde{c}_{1}\right) \neq 0$ one gets $\operatorname{Re}\left(c_{n}\right)=\operatorname{Re}\left(\tilde{c}_{n}\right)$, completing the proof.

Q.E.D.

Acknowledgements. The author would like to thank Walter Neumann for his encouragement and helpful e-mail correspondence. This work is supported in part by the Rackham Faculty Grant and Fellowship at the University of Michigan.

\section{References}

[AR] I. Aitchison, H. Rubinstein: Combinatorial Cubings, cusps, and the dodecahegral knots. Topology'90, Proceedings of the Research Semester on Low Dimensional Topology at Ohio State. Walter de Gruyter Verlag, 1992, 17-26

[EP] D. Epstein, R. Penner: Euclidean decompositions of non-compact hyperbolic manifolds. J. Diff. Geom. 27 (1988), 67-80

[NR1] W. Neumann, A. Reid: Arithmetic of hyperbolic manifolds. Topology'90, Proceedings of the Research Semester on Low Dimensional Topology at Ohio State. Walter de Gruyter Verlag, 1992, 273-310

[NR2] W. Neumann, A. Reid: Amalgamations and the invariant trace field. Math. Proc. Camb. Philos. Soc. 109 (1991), 509-515

[NZ] W. Neumann, D. Zagier: Volumes of hyperbolic 3-manifolds. Topology 24 (1985), 307-332

[Ro] D. Rolfsen: Knots and Links. Publish or Perish, 1990

[Ru] D. Ruberman: Mutation and volumes of knots in $S^{3}$. Invent. Math. 90 (1987), $189-215$

[T] W. Thurston: The geometry and topology of 3-manifolds. Lecture notes. Princeton University, 1978

[Y] T. Yoshida: The $\eta$-invariant of hyperbolic 3-manifolds. Invent. Math. 81 (1985), $473-514$ 